Journal of Computer Science 3 (9): 740-746, 2007

ISSN 1549-3636

(C) 2007 Science Publications

\title{
Combined DWT-DCT Digital Image Watermarking
}

\author{
Ali Al-Haj \\ Department of Computer Engineering, School of Electrical Engineering, \\ Princess Sumaya University for Technology, PO Box 1928, Al-Jubeiha, \\ 11941 Amman, Jordan
}

\begin{abstract}
The proliferation of digitized media due to the rapid growth of networked multimedia systems, has created an urgent need for copyright enforcement technologies that can protect copyright ownership of multimedia objects. Digital image watermarking is one such technology that has been developed to protect digital images from illegal manipulations. In particular, digital image watermarking algorithms which are based on the discrete wavelet transform have been widely recognized to be more prevalent than others. This is due to the wavelets' excellent spatial localization, frequency spread, and multi-resolution characteristics, which are similar to the theoretical models of the human visual system. In this paper, we describe an imperceptible and a robust combined DWT-DCT digital image watermarking algorithm. The algorithm watermarks a given digital image using a combination of the Discrete Wavelet Transform (DWT) and the Discrete Cosine Transform (DCT). Performance evaluation results show that combining the two transforms improved the performance of the watermarking algorithms that are based solely on the DWT transform.
\end{abstract}

Key words: Digital image watermarking, image copyright protection, frequency-domain watermarking, Discrete Wavelet Transform (DWT), Discrete Cosine Transform (DCT)

\section{INTRODUCTION}

The development of effective digital image copyright protection methods have recently become an urgent and necessary requirement in the multimedia industry due to the ever-increasing unauthorized manipulation and reproduction of original digital objects. The new technology of digital watermarking has been advocated by many specialists as the best method to such multimedia copyright protection problem $^{[1,2]}$. Its expected that digital watermarking will have a wide-span of practical applications such as digital cameras, medical imaging, image databases, and video-on-demand systems, among many others ${ }^{[3]}$.

In order for a digital watermarking method to be effective it should be imperceptible, and robust to common image manipulations like compression, filtering, rotation, scaling cropping, collusion attacks among many other digital signal processing operations. Current digital image watermarking techniques can be grouped into two major classes: spatial-domain and frequency-domain watermarking techniques ${ }^{[4]}$. Compared to spatial domain techniques ${ }^{[5]}$, frequency-domain watermarking techniques proved to be more effective with respect to achieving the imperceptibility and robustness requirements of digital watermarking algorithms ${ }^{[6]}$.

Commonly used frequency-domain transforms include the Discrete Wavelet Transform (DWT), the Discrete Cosine Transform (DCT) and Discrete Fourier Transform (DFT). However, $\mathrm{DWT}^{[7]}$ has been used in digital image watermarking more frequently due to its excellent spatial localization and multi-resolution characteristics, which are similar to the theoretical models of the human visual system ${ }^{[8]}$. Further performance improvements in DWT-based digital image watermarking algorithms could be obtained by combining DWT with $\mathrm{DCT}^{[9]}$. The idea of applying two transform is based on the fact that combined transforms could compensate for the drawbacks of each other, resulting in effective watermarking.

In this paper, we will describe a digital image watermarking algorithm based on combining two transforms; DWT and DCT. Watermarking is done by altering the wavelets coefficients of carefully selected DWT sub-bands, followed by the application of the DCT transform on the selected sub-bands.

Corresponding Author: Ali Al-Haj, Department of Computer Engineering, School of Electrical Engineering, Princess Sumaya University for Technology, PO Box 1928, Al-Jubeiha, 11941 Amman, Jordan 


\section{THE DCT AND DWT TRANSFORMS}

The DCT and DWT transforms have been extensively used in many digital signal processing applications. In this section, we introduce the two transforms briefly, and outline their relevance to the implementation of digital watermarking.

The DCT transform: The discrete cosine transforms is a technique for converting a signal into elementary frequency components ${ }^{[9]}$. It represents an image as a sum of sinusoids of varying magnitudes and frequencies. With an input image, $\mathrm{x}$, the DCT coefficients for the transformed output image, $y$, are computed according to Eq. 1 shown below. In the equation, $\mathrm{x}$, is the input imagehaving $\mathrm{N} x \mathrm{M}$ pixels, $\mathrm{x}(\mathrm{m}, \mathrm{n})$ is the intensity of the pixel in row $\mathrm{m}$ and column $\mathrm{n}$ of the image, and $\mathrm{y}(\mathrm{u}, \mathrm{v})$ is the DCT coefficient in row $\mathrm{u}$ and column $\mathrm{v}$ of the DCT matrix.

$$
\begin{aligned}
& y(u, v)=\sqrt{\frac{2}{M}} \sqrt{\frac{2}{N}} \alpha_{u} \alpha_{v} \sum_{u=0}^{M-1} \sum_{v=0}^{N-1} x(m, n) \\
& \cos \frac{(2 m+1) u \Pi}{2 M} \cos \frac{(2 n+1) v \Pi}{2 N}
\end{aligned}
$$

where

$$
\begin{aligned}
& \alpha_{u}= \begin{cases}\frac{1}{\sqrt{2}} & u=0 \\
1 & u=1,2, \ldots, N-1\end{cases} \\
& \alpha_{v}= \begin{cases}\frac{1}{\sqrt{2}} & \mathrm{v}=0 \\
1 & \mathrm{v}=1,2, \ldots, \mathrm{N}-1\end{cases}
\end{aligned}
$$

The image is reconstructed by applying inverse DCT operation according to Eq. 2:

$$
\begin{aligned}
& x(m, n)=\sqrt{\frac{2}{M}} \sqrt{\frac{2}{N}} \sum_{u=0}^{M-1} \sum_{v=0}^{N-1} \alpha_{u} \alpha_{v} y(u, v) \\
& \cos \frac{(2 m+1) u \prod}{2 M} \cos \frac{(2 n+1) v \prod}{2 N}
\end{aligned}
$$

The popular block-based DCT transform segments an image non-overlapping blocks and applies DCT to each block. This results in giving three frequency sub-bands: low frequency sub-band, mid-frequencysub-ban and high frequency sub-band. DCT-based watermarking is based on two facts. The first fact is that much of the signal energy lies at low-frequencies sub-band which contains the most important visual parts of the image. The second fact is that high frequency components of the image are usually removed through compression and noise attacks. The watermark is therefore embedded by modifying the coefficients of the middle frequency sub-band so that the visibility of the image will not be affected and the watermark will not be removed by compression $^{[10,11,12,13]}$.

The DWT transform: Wavelets are special functions which, in a form analogous to sines and cosines in Fourier analysis, are used as basal functions for representing signals ${ }^{[7]}$. For 2-D images, applying DWT corresponds to processing the image by 2-D filters in each dimension. The filters divide the input image into four non-overlapping multi-resolution sub-bands LL1, LH1, HL1 and HH1. The sub-band LL1 represents the coarse-scale DWT coefficients while the sub-bands LH1, HL1 and HH1 represent the fine-scale of DWT coefficients. To obtain the next coarser scale of wavelet coefficients, the sub-band LL1 is further processed until some final scale $\mathrm{N}$ is reached. When $\mathrm{N}$ is reached we will have $3 \mathrm{~N}+1$ sub-bands consisting of the multi-resolution sub-bands $\mathrm{LL}_{\mathrm{N}}$ and LHx, HLx and $\mathrm{HHx}$ where $\mathrm{x}$ ranges from 1 until $\mathrm{N}$.

Due to its excellent spatio-frequency localization properties, the DWT is very suitable to identify the areas in the host image where a watermark can be embedded effectively. In particular, this property allows the exploitation of the masking effect of the human visual system such that if a DWT coefficient is modified, only the region corresponding to that coefficient will be modified. In general most of the image energy is concentrated at the lower frequency sub-bands LLx and therefore embedding watermarks in these sub-bands may degrade the image significantly. Embedding in the low frequency sub-bands, however, could increase robustness significantly. On the other hand, the high frequency sub-bands $\mathrm{HHx}$ include the edges and textures of the image and the human eye is not generally sensitive to changes in such sub-bands. This allows the watermark to be embedded without being perceived by the human eye. The compromise adopted by many DWT-based watermarking algorithm, is to embed the watermark in the middle frequency ub-bands LHx and HLx where acceptable performance of imperceptibility and robustness could be achieved ${ }^{[14,15,16,17,18,19,20]}$. 


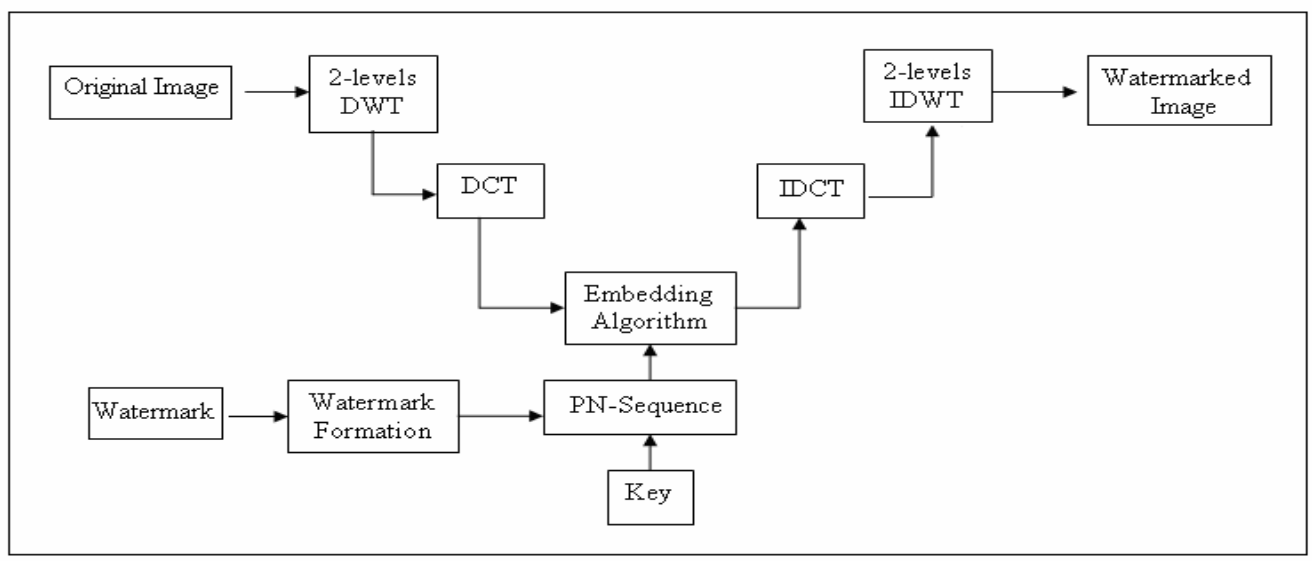

Fig. 1: Combined DWT-DCT watermark embedding procedure.

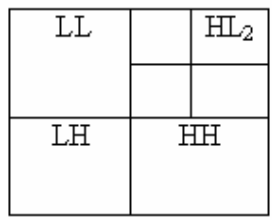

(a)

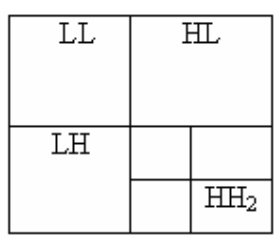

(b)
Fig. 2: Multi-resolution DWT sub-bands of the original image

\section{THE COMBINED DCT-DWT ALGORTIHM}

The watermark embedding procedure is depicted in Fig. 1 followed by a detailed explanation.

Step 1: Apply DWT to decompose the cover host image into four non-overlapping multi-resolution sub-bands: $\mathrm{LL}_{1}, \mathrm{HL}_{1}, \mathrm{LH}_{1}$, and $\mathrm{HH}_{1}$.

Step 2: Apply DWT again to sub-band $\mathrm{HL}_{1}$ to get four smaller sub-bands and choose the $\mathrm{HL}_{2}$ sub-band as shown in Fig. 2 a. Or, apply DWT to sub-band $\mathrm{HH}_{1}$ to get four smaller sub-bands and choose the $\mathrm{HH}_{2}$ sub-band as shown in Fig. 2 b.

Step 3: Divide the sub-band $\mathrm{HL}_{2}\left(\right.$ or $\left.\mathrm{HH}_{2}\right)$ into $4 \times 4$ blocks.

Step 4: Apply DCT to each block in the chosen sub-band $\left(\mathrm{HL}_{2}\right.$ or $\left.\mathrm{HH}_{2}\right)$.

Step 5: Re-formulate the grey-scale watermark image into a vector of zeros and ones.
Step 6: Generate two uncorrelated pseudorandom sequences. One sequence is used to embed the watermark bit 0 (PN_0) and the other sequence is sued to embed the watermark bit 1 (PN_1). Number of elements in each of the two pseudorandom sequences must be equal to the number of mid-band elements of the DCT-transformed DWT sub-bands.

Step 7: Embed the two pseudorandom sequences, PN_0 and PN_1, with a gain factor $\alpha$, in the DCT transformed $4 \times 4$ blocks of the selected DWT sub-bands of the host image.

Embedding is not applied to all coefficients of the DCT block, but only to the mid-band DCT coefficients. If we donate $\boldsymbol{X}$ as the matrix of the midband coefficients of the DCT transformed block, then embedding is done as follows:

If the watermark bit is 0 then

$$
\mathrm{X}^{\prime}=\mathrm{X}+\alpha^{*} \mathrm{PN} \_0
$$

otherwise,

if the watermark bit is 1 then,

$$
X^{\prime}=X+\alpha * P N \_1
$$

Step 8: Apply inverse DCT (IDCT) to each block after its mid-band coefficients have been modified to embed the watermark bits as described in the previous step.

Step 9: Apply the inverse DWT (IDWT) on the DWT transformed image, including the modified sub-band, to produce the watermarked host image.

The watermark extraction procedure is depicted in Fig. 3, and described in details in the following steps. The combined DWT-DCT algorithm is a blind 


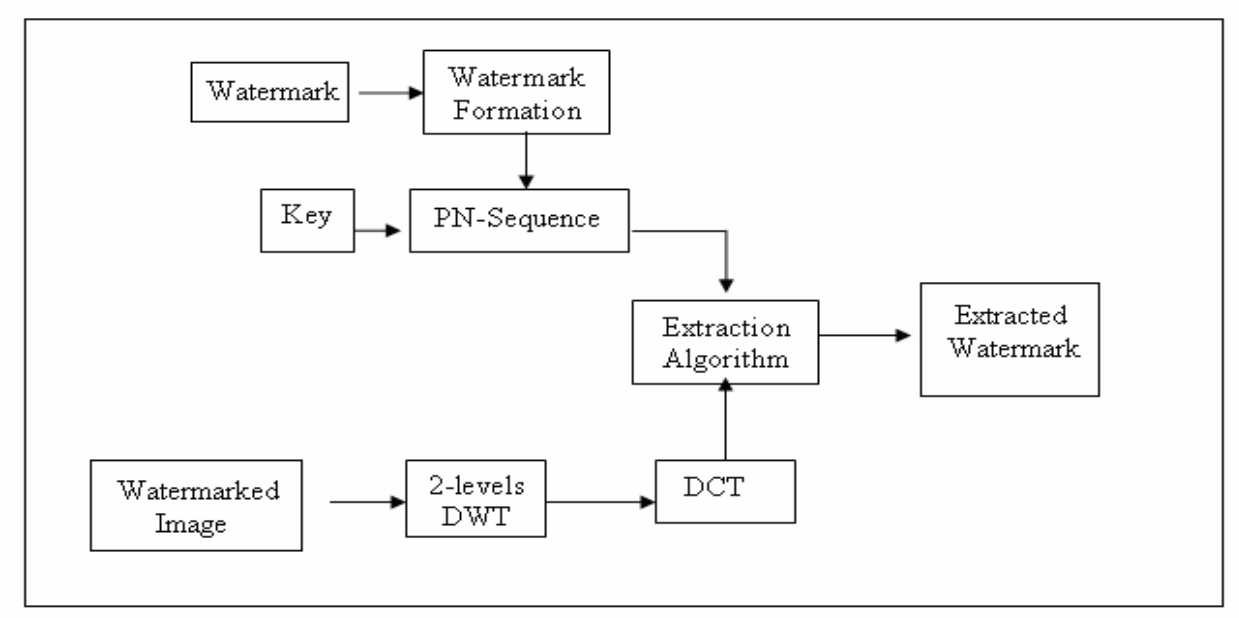

Fig. 3: Combined DWT-DCT watermark extraction procedure

watermarking algorithm, and thus the original host image is not required to extract the watermark.

Step 1: Apply DWT to decompose the watermarked image into four non-overlapping multi-resolution subbands: $\mathrm{LL}_{1}, \mathrm{HL}_{1}, \mathrm{LH}_{1}$, and $\mathrm{HH}_{1}$.

Step 2: Apply DWT to $\mathrm{HL}_{1}$ to get four smaller subbands, and choose the sub-band $\mathrm{HL}_{2}$, as shown in Fig. 2 a. Or, apply DWT to the $\mathrm{HH}_{1}$ sub-band to get four smaller sub-bands, and choose the $\mathrm{HH}_{2}$ sub-band, as shown in Fig. 2 b.

Step 3: Divide the sub-ban $\mathrm{HL}_{2}$ (or $\mathrm{HH}_{2}$ ) into $4 \times 4$ blocks.

Step 4: Apply DCT to each block in the chosen sub-band $\left(\mathrm{HL}_{2}\right.$ or $\left.\mathrm{HH}_{2}\right)$, and extract the mid-band coefficients of each DCT transformed block.

Step 5: Regenerate the two pseudorandom sequences (PN_0 and PN_1) using the same seed used in the watermark embedding procedure.

Step 6: For each block in the sub-band $\mathrm{HL}_{2}\left(\right.$ or $\left.\mathrm{HH}_{2}\right)$, calculate the correlation between the mid-band coefficients and the two generated pseudorandom sequences (PN_0 and PN_1). If the correlation with the PN_0 was higher than the correlation with PN_1, then the extracted watermark bit is considered 0 , otherwise the extracted watermark is considered 1.

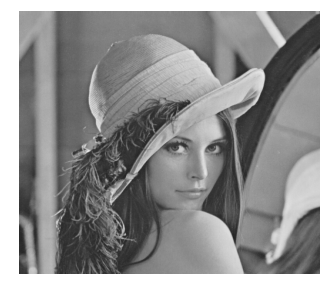

Fig. 4: 'Lina' host image

\section{Copyright}

Fig. 5: Original watermark

Step 7: Reconstruct the watermark using the extracted watermark bits, and compute the similarity between the original and extracted watermarks.

\section{PERFORMANCE EVALUATION}

We evaluated the performance of the combined DWT-DCT image watermarking algorithms using a $512 \times 512$ 'Lena' as the original cover host image, and a $256 \times 256$ grey-scale image of the expression 'copyright' as the watermark image. The two images are shown in Fig. 4 and 5, respectively.

Performance Evaluation Metrics: Watermarking algorithms are usually evaluated with respect to two metrics: imperceptibility and robustness ${ }^{[21]}$. The two metrics are described below. 
Table 1: The 'Lina' host image watermarked at different 2-DWT sub-bands

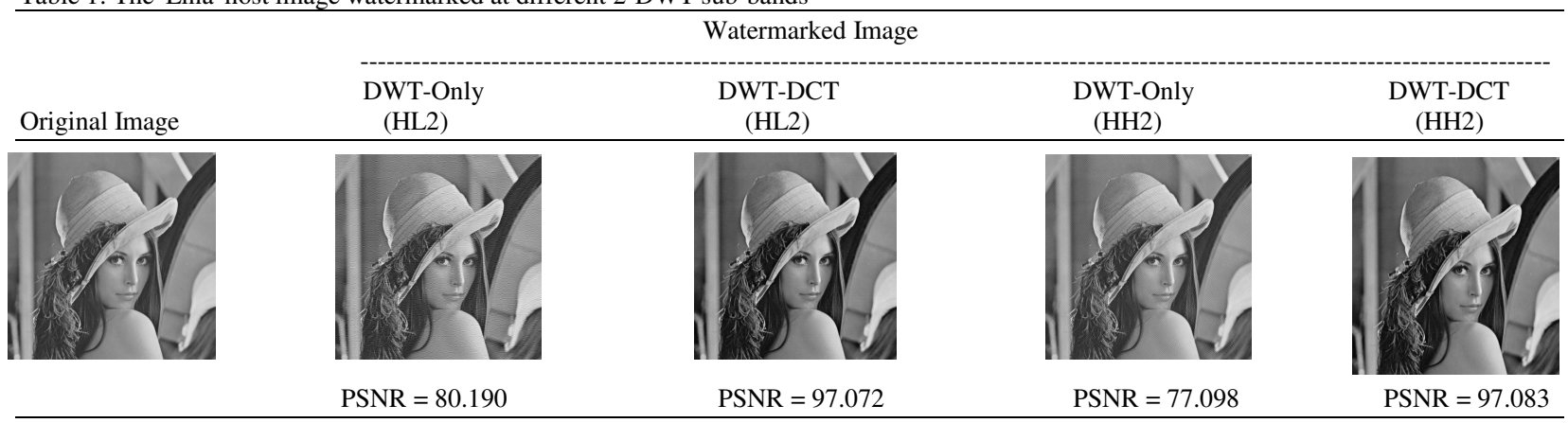

Imperceptibility: Imperceptibility means that the perceived quality of the host image should not be distorted by the presence of the watermark. As a measure of the quality of a watermarked image, the peak signal to noise ratio (PSNR) is typically used. PSNR in decibels $(\mathrm{dB})$ is given below in Eq. 5 .

$$
\begin{aligned}
& \operatorname{PSNR}_{\mathrm{dB}}=10 \cdot \log _{10}\left(\frac{\mathrm{MAX}_{\mathrm{I}}^{2}}{\mathrm{MSE}}\right) \\
& =20 \cdot \log _{10}\left(\frac{\mathrm{MAX}_{\mathrm{I}}}{\sqrt{\mathrm{MSE}}}\right)
\end{aligned}
$$

Robustness: Robustness is a measure of the immunity of the watermark against attempts to remove or degrade it, internationally or unintentionally, by different types of digital signal processing attacks ${ }^{\text {[22] }}$. In this chapter, we will report on robustness results which we obtained for three major digital signal processing operations (attacks): Gaussian noise, image compression and image cropping. The three attacks are a few, however, they are good representatives of the more general attacks. That is the Gaussian noise is a watermark degrading attack, JPEG compression is a watermark removal attack and cropping is a watermark dispositioning geometrical attack. We measured the similarity between the original watermark and the watermark extracted from the attacked image using the correlation factor $\rho$ given below in Eq. 6 .

$$
\rho(w, \hat{w})=\frac{\sum_{i=1}^{N} w_{i} \hat{w}_{i}}{\sqrt{\sum_{i=1}^{N} w_{i}^{2}} \sqrt{\sum_{i=1}^{N} \hat{w}_{i}^{2}}}
$$

where $\mathrm{N}$ is the number of pixels in watermark, $\mathrm{w}$ and $\hat{\mathrm{w}^{\wedge}}$ are the original and extracted watermarks respectively. The correlation factor $\rho$ may take values between 0 (random relationship) to 1 (perfect linear relationship). In general, a correlation coefficient of about 0.75 or above is considered acceptable.

Performance results: We carried out the watermark embedding algorithm described in the previous section. Applying the 1-level DWT of the 'Lina' image produced four 256256 sub-bands; $\mathrm{LL}_{1}, \mathrm{LH}_{1}, \mathrm{HL}_{1}$ and $\mathrm{HH}_{1}$. Since embedding the watermark beyond the first DWT level is more effective, we decided to embed the watermark in $\mathrm{HL}_{2}$ (or $\mathrm{HH}_{2}$ ). The selected $128 \times 128$ sub-band was divided into $4 \times 4$ blocks giving a total of 1024 blocks. The DCT transform was then applied to each block in the chosen sub-band, after which the watermark was embedded according to Eq. 3 and 4.

Imperceptibility: We evaluated imperceptibility of the combined DWT-DCT algorithm by measuring PSNR for the $\mathrm{HL}_{2}$ and $\mathrm{HH}_{2}$ sub-bands. $\mathrm{HL}_{2}$ gave a PSNR value of 97.072 while $\mathrm{HH}_{2}$ gave 97.083. The difference is small between the two PSNR values indicating comparable performance of the two sub-bands. In contrast, and as shown in Table 1, the difference between the PSNR values of the DWT-Only and the combined DWT-DCT algorithms is relatively large. This indicates that improvement in imperceptibility can be achieved by applying DCT on a DWT transformed $\mathrm{HL}_{2}$ (or $\mathrm{HH}_{2}$ ) sub-band.

Robustness: Table 2 shows the correlation values between the original watermark and the watermarks extracted from sub-bands $\mathrm{HL}_{2}$ and $\mathrm{HH}_{2}$ after being subjected to different attacks, independently.

The correlation values given in Table 2 show clearly that the combined DWT-DCT watermarking algorithm outperforms the conventional DWT-Only approach with respect to robustness against the Gaussian noise and cropping attacks. The results are better regardless of whether the watermark was embedded in $\mathrm{HL}_{2}$ or $\mathrm{HH}_{2}$, however, $\mathrm{HH}_{2}$ gave better 
Table 2: Correlation values due to three different attacks

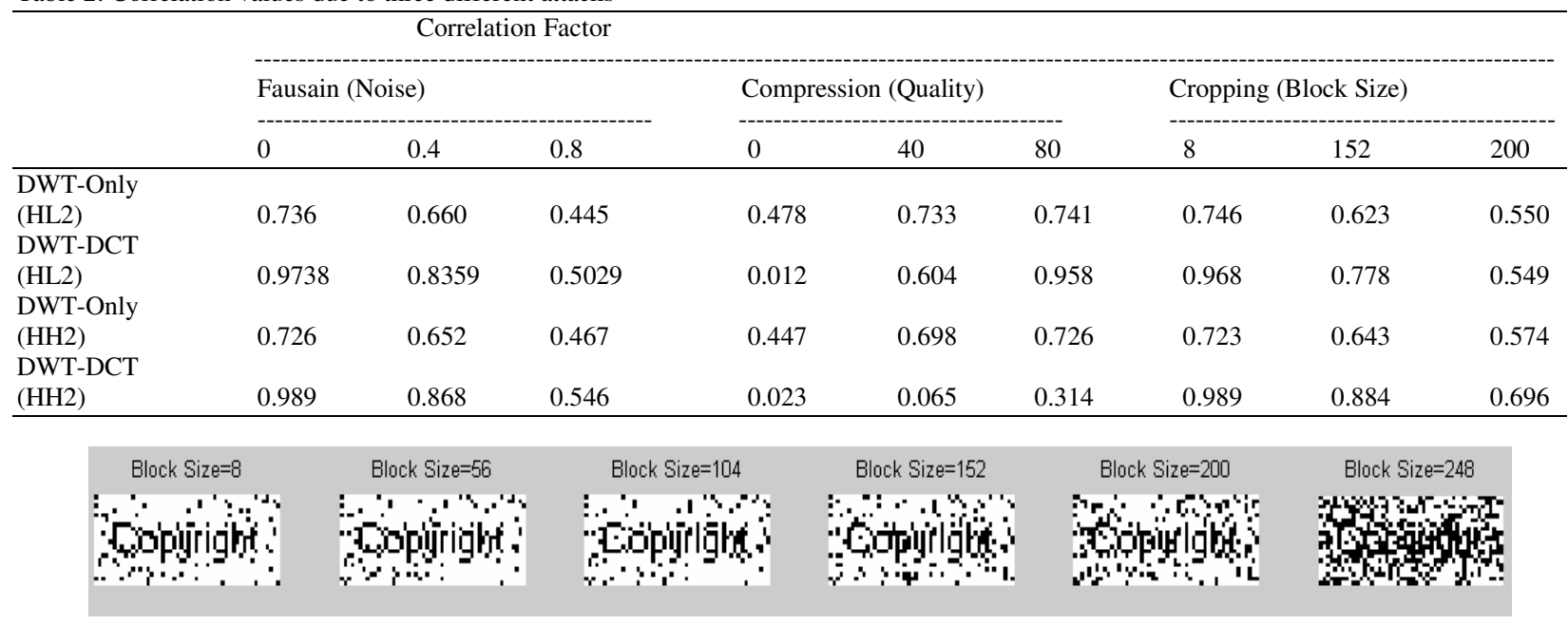

Fig. 6: Extracted watermarks from the cropped HH2-watermarked image using DWT only

\begin{tabular}{|c|c|c|c|c|c|}
\hline Block Size=8 & Block Size=56 & Block Size $=104$ & Block Size=152 & Block Size $=200$ & Block Size $=248$ \\
\hline Copplight & & & "ו & & $\therefore$ \\
\hline
\end{tabular}

Fig. 7: Extracted watermarks from the cropped HH2-wateramrked image using DWT and DCT

Quality

Fig. 8: Extracted watermarks from the compressed HL2-watermarked image using DWT only

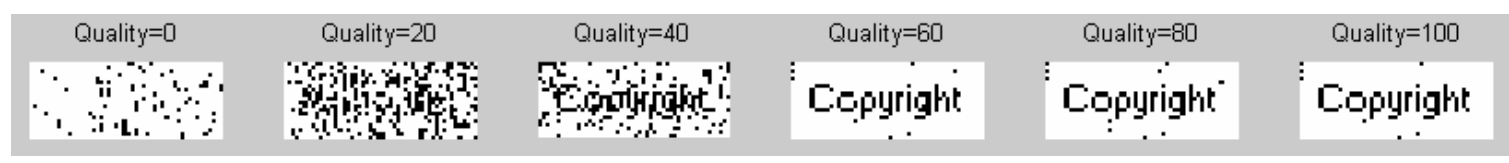

Fig. 9: Extracted watermarks from the compressed HL2-watermarked image using DWT and DCT

robustness against cropping compared with $\mathrm{HL}_{2}$. Figure 6 shows the extracted watermarks from $\mathrm{HH}_{2}$ when DWT-Only was used. In contrast, the better combined DWT-DCT results are shown in Figure 7 which shows the extracted watermarks from the $\mathrm{HH}_{2}$ sub-band.

As given in Table 2 and illustrated in Fig. 8 and 9, the robustness of the DWT-Only approach against the JPEG compression attack is better than that of the combined DWT-DCT algorithm. This is due to the fact that the DCT is the core component of the JPEG compression, and therefore applying DCT at a DCTtransformed image degraded the quality of the embedded watermark greatly.

\section{RESULTS AND DISCUSSION}

We described the performance of the combined DWT-DCT watermarking algorithm. For the sake of comparison, we also evaluated the watermarking performance when DWT-Only was used. The results we obtained for the DWT-Only approach indicated a better imperceptibility performance was obtained when the watermark was embedded in the $\mathrm{HL}_{2}$ or $\mathrm{HH}_{2}$ subbands. The robustness performance, however, was not acceptable. To improve performance, we combined DWT with the another equally powerful transform; the DCT. The combined DWT-DCT watermarking algorithm's imperceptibility performance was better 
than the performance of the DWT-Only approach. Moreover, the improvement in robustness brought by the combined DWT-DCT algorithm was considerably high.

\section{CONCLUSIONS}

The discrete wavelet transform (DWT) and the discrete cosine transform (DCT) have been applied successfully in many in digital image watermarking. In this paper, we described a combined DWT-DCT digital image watermarking algorithm. Watermarking was done by embedding the watermark in the first and second level DWT sub-bands of the host image, followed by the application of DCT on the selected DWT sub-bands. The combination of the two transforms improved the watermarking performance considerably when compared to the DWT-Only watermarking approach. In conclusion, in DWT-based digital watermarking applications, combining appropriate transforms with the DWT may have a positive impact on performance of the watermarking system.

\section{REFERENCES}

1. Cox, I., M. Miller and J. Bloom, 2002. Digital Watermarking, Academic Press, USA.

2. Langelaar, G., I. Setyawan and R. Lagendijk, 2000. "Watermarking Digital Image and Video Data: A State-of-Art Overview," IEEE Signal Processing Magazine, 17(5):20-46.

3. Arnold, M., M. Schumucker and S. Wolthusen, 2003. Techniques and Applications of Digital Watermarking and Content Protection. Artech House, USA.

4. Potdar, V., S. Han and E. Chang, 2005. A Survey of Digital Image Watermarking Techniques, in Proc. of the IEEE International Conference on Industrial Informatics, pp: 709-716, Perth, Australia.

5. Chan, C. and L. Cheng, 2004. Hiding Data in Images by Simple LSB Substitution, Pattern Recognition, 37(3):469-474.

6. Wang, R., C. Lin and J. Lin, " Copyright protection of digital images by means of frequency domain watermarking," Proc. of the SPIE Conference On Mathematics of Data/Image Coding, Compression, and Encryption, USA.

7. Vetterli, M. and J. Kovačević, 1995. Wavelets and Subband Coding. Prentice Hall, USA.

8. Wolfgang, R., C. Podilchuk and E. Delp, 1999. "Perceptual Watermarks for Digital Images and Video," Proc. of the IEEE, vol. 87, no. 7, pp: 1108-1126.
9. Rao, K. and P. Yip. Discrete Cosine Transform: algorithms, advantages, applications. Academic Press, USA, 1990.

10. Chu, W, 2003. "DCT-Based Image Watermarking Using Subsampling," IEEE Trans. Multimedia, 5(1): 34-38.

11. Lin, S. and C. Chin, 2000. "A Robust DCT-based Watermarking for Copyright Protection," IEEE Trans. Consumer Electronics, 46(3): 415-421.

12. Deng, F. and B. Wang, 2003. "A novel technique for robust image watermarking in the DCT domain," in Proc. of the IEEE 2003 Int. Conf. on Neural Networks and Signal Processing, vol. 2, pp: 1525-1528.

13. Wu, C. and W. Hsieh, 2000. "Digital watermarking using zerotree of DCT," IEEE Trans. Consumer Electronics, vol. 46, no. 1, pp: 87-94.

14. Hsieh, M., D. Tseng, and Y. Huang, 2001. "Hiding Digital Watermarks Using Multiresolution Wavelet Transform," IEEE Trans. on Industrial Electronics, 48(5): 875-882.

15. Reddy, A. and B. Chatterji, 2005. "A New Wavelet Based Logo-watermarking Scheme," Pattern Recognition Letters, 26(7): 1019-1027.

16. Wang, S. and Y. Lin, 2004. "Wavelet Tree Quantization for Copyright Protection Watermarking," IEEE Trans. Image Processing, vol. 13, no. 2, pp: 154-164.

17. Hsu, C. and J. Wu, 1998. "Multiresolution Watermarking for Digital Images," IEEE Trans. on Circuits and Systems- II, 45(8): 1097-1101.

18. Tay, P. and J. Havlicek, 2002. "Image Watermarking Using Wavelets," in Proc. of the IEEE Midwest Symposium on Circuits and Systems, pp: 258-261, Oklahoma, USA.

19. Nikolaidis, A. and I. Pitas, 2003. "Asymptotically optimal detection for additive watermarking in the DCT and DWT domains," IEEE Trans. Image Processing , 2(10): 563-571.

20. Tsai, M. and H. Hung, 2005. "DCT and DWTbased Image Watermarking Using Subsampling," in Proc. of the 2005 IEEE Fourth Int. Conf. on Machine Learning and Cybernetics, pp: 5308-5313, China.

21. Ejima, M. and A. Myazaki, 2001." On the evaluation of performance of digital watermarking in the frequency domain," in Proc. of the IEEE Int. Conf. on Image Processing, 2: 546-549.

22. Voloshynovskiy, S., S. Pereira and T. Pun, 2001. "Attacks on Digital Watermarks: Classification, Estimation-Based Attacks, and Benchmarks," Comm. Magazine, 39(8): 118-126. 